\title{
Coronary calcium mass scores measured by identical 64-slice MDCT scanners are comparable: a cardiac phantom study
}

\author{
Hildebrand Dijkstra · Marcel J. W. Greuter · Jaap M. Groen • \\ Rozemarijn Vliegenthart-Proença · KlaasJan W. K. Renema • \\ Frank de Lange $\cdot$ Matthijs Oudkerk
}

Received: 8 June 2009/ Accepted: 25 August 2009/Published online: 19 September 2009

(C) The Author(s) 2009. This article is published with open access at Springerlink.com

\begin{abstract}
To assess whether absolute mass scores are comparable or differ between identical 64-slice MDCT scanners of the same manufacturer and to compare absolute mass scores to the physical mass and between scan modes using a calcified phantom. A non-moving anthropomorphic phantom with nine calcifications of three sizes and three densities was scanned 30 times on three 64-slice MDCT scanners of manufacturer $\mathrm{A}$ and on three 64-slice MDCT scanners of manufacturer $\mathrm{B}$ in both sequential and spiral scan mode. The mean mass scores and mass score variabilities of seven calcifications were determined for all scanners; two non-detectable calcifications were omitted. It was analyzed whether identical scanners yielded similar or significantly different mass scores. Furthermore mass scores were compared to the physical mass and mass scores were compared between scan modes. The mass score calibration factor was determined for all scanners. Mass scores obtained on identical scanners were similar for almost all calcifications. Overall, mass score
\end{abstract}

H. Dijkstra ( $\varangle)$ · M. J. W. Greuter · J. M. Groen ·

R. Vliegenthart-Proença $\cdot$ M. Oudkerk

Department of Radiology, University Medical Center

Groningen, Groningen, The Netherlands

e-mail: h.dijkstra@rad.umcg.nl

K. W. K. Renema · F. de Lange

Department of Radiology, University Medical Center St

Radboud, Nijmegen, The Netherlands differences between the scanners were small ranging from 1.5 to $3.4 \%$ for the total mass scores, and most differences between scanners were observed for high density calcifications. Mass scores were significantly different from the physical mass for almost all calcifications and all scanners. In sequential mode the total physical mass $(167.8 \mathrm{mg}$ ) was significantly overestimated $(+2.3 \%)$ for 4 out of 6 scanners. In spiral mode a significant overestimation $(+2.5 \%)$ was found for system B and a significant underestimation $(-1.8 \%)$ for two scanners of system A. Mass scores were dependent on the scan mode, for manufacturer A scores were higher in sequential mode and for manufacturer B in spiral mode. For system A using spiral scan mode no differences were found between identical scanners, whereas a few differences were found using sequential mode. For system B the scan mode did not affect the number of different mass scores between identical scanners. Mass scores obtained in the same scan mode are comparable between identical 64-slice CT scanners and identical 64-slice CT scanners on different sites can be used in follow-up studies. Furthermore, for all systems significant differences were found between mass scores and the physical calcium mass; however, the differences were relatively small and consistent.

Keywords Coronary artery calcification .

Calcium mass score - Inter-scanner variability . MDCT · Computed tomography · Myocardial event . Calcium score $\cdot$ Calcium quantification 


\section{Introduction}

New computed tomography techniques enable the detection and quantification of coronary calcification. In 1990 a scoring algorithm was proposed by Agatston to quantify the amount of calcium in the coronary arteries, using electron beam CT (EBT) [1]. In large and diverse studies the Agatston score has been found to be a strong predictor of future myocardial events [2-5]. However, because of the limited reproducibility of the calcium score according to Agatston, other scoring algorithms were introduced, like the volume score and the mass score [6-8]. Various studies showed the lower variability of the mass score compared to Agatston score and volume score [9-13].

Currently Multi-Detector CT (MDCT) systems are widely used to diagnose and quantify coronary calcifications. A large number of medical centers have multiple MDCT systems installed and multisite studies assessing calcium scores are performed more and more frequently $[14,15]$. The number of patients examined on different scanners for followup calcium score determination is therefore expected to increase. It is important to understand the effect of using different scanners in consecutive calcium score determinations of the same patient, particularly when the calcium score is used as a marker to follow the development of atherosclerosis. Then, it is especially important to establish whether a difference in calcium score reflects a true change in calcium or is due to interscan variability or due to a difference in scan technique. Several articles have proposed repeatability limits based on EBT and on MDCT to define significant change of coronary calcium on repeated scans $[15,16]$. Calcium score protocols for MDCT are not standardized and can be performed using both sequential and spiral acquisition modes.

The purpose of this study was therefore threefold. First we assessed whether absolute mass scores are comparable or differ between identical 64-slice MDCT scanners of the same manufacturer, and determined mass score variability. Secondly, it was determined how the mass scores compared to the physical mass. And finally the effect of the scan mode on the absolute mass scores was analyzed. Three identical scanners of manufacturer $\mathrm{A}$ and of manufacturer B were included.

\section{Materials and methods}

A non-moving anthropomorphic phantom (QRM, Möhrendorf, Germany) with nine calcifications of three sizes (1, 3 and $5 \mathrm{~mm}$ ) and three densities (low, medium and high) was scanned (Fig. 1). The phantom was scanned with three 64-slice MDCT scanners of manufacturer A (system A) and three of manufacturer B (system B). On each 64-slice MDCT scanner the measurements were performed with a sequential and a spiral protocol. For each scanner and scan mode, the phantom was scanned 30 times. In between the consecutive scans, the phantom was randomly dislocated by shifting it several millimeters and rotating it a few degrees in the horizontal plane, in order to simulate the random positioning of the coronaries in each $\mathrm{R}-\mathrm{R}$ interval. The phantom was also scanned fifteen times without random movement for each scanner and scan mode.

The scan parameters on system A were: tube voltage $120 \mathrm{kV}$, collimation $64 \times 0.6 \mathrm{~mm}$ and rotation time $330 \mathrm{~ms}$. System B was used with similar scan parameters: tube voltage $120 \mathrm{kV}$, collimation $64 \times 0.5 \mathrm{~mm}$ and rotation time $400 \mathrm{~ms}$. Tube currents were $50 \mathrm{mAs}$ in sequential scan mode for all scanners. For the spiral acquisitions the tube currents were $110 \mathrm{mAs}$ for the scanners of system $\mathrm{A}$ and $76 \mathrm{mAs}$ for the scanners of system B.

For both systems the acquired data were reconstructed at $75 \%$ of the R-R interval with nonoverlapping $3 \mathrm{~mm}$ slice thickness and $320 \mathrm{~mm}$ FOV. For ECG gating an ECG signal was generated by an external patient simulator with a frequency of $71 \mathrm{bpm}$. Reconstruction was performed using a similar medium smooth convolution kernel available on both systems.

Reconstructed images were analyzed on a Siemens Syngo workstation (Siemens, Forchheim, Germany) using the application $\mathrm{Ca}$ score. First, the mass score was calibrated on each scanner to ensure that mass scores of the individual scanners could be compared. Calibration was performed by measuring two calibration inserts with known densities available in the QRM phantom; a water equivalent insert (0 HU) and a medium density insert of $200 \mathrm{HU}$ (Fig. 1). Subsequently the calibration factor was calculated as described in the literature [9] (Table 1). Finally the mass score $M S(\mathrm{mg})$ was determined for all individual calcifications above the default threshold of $130 \mathrm{HU}$. 
Fig. 1 QRM heart insert with nine calcifications (top) as part of the anthropomorphic phantom body (bottom)
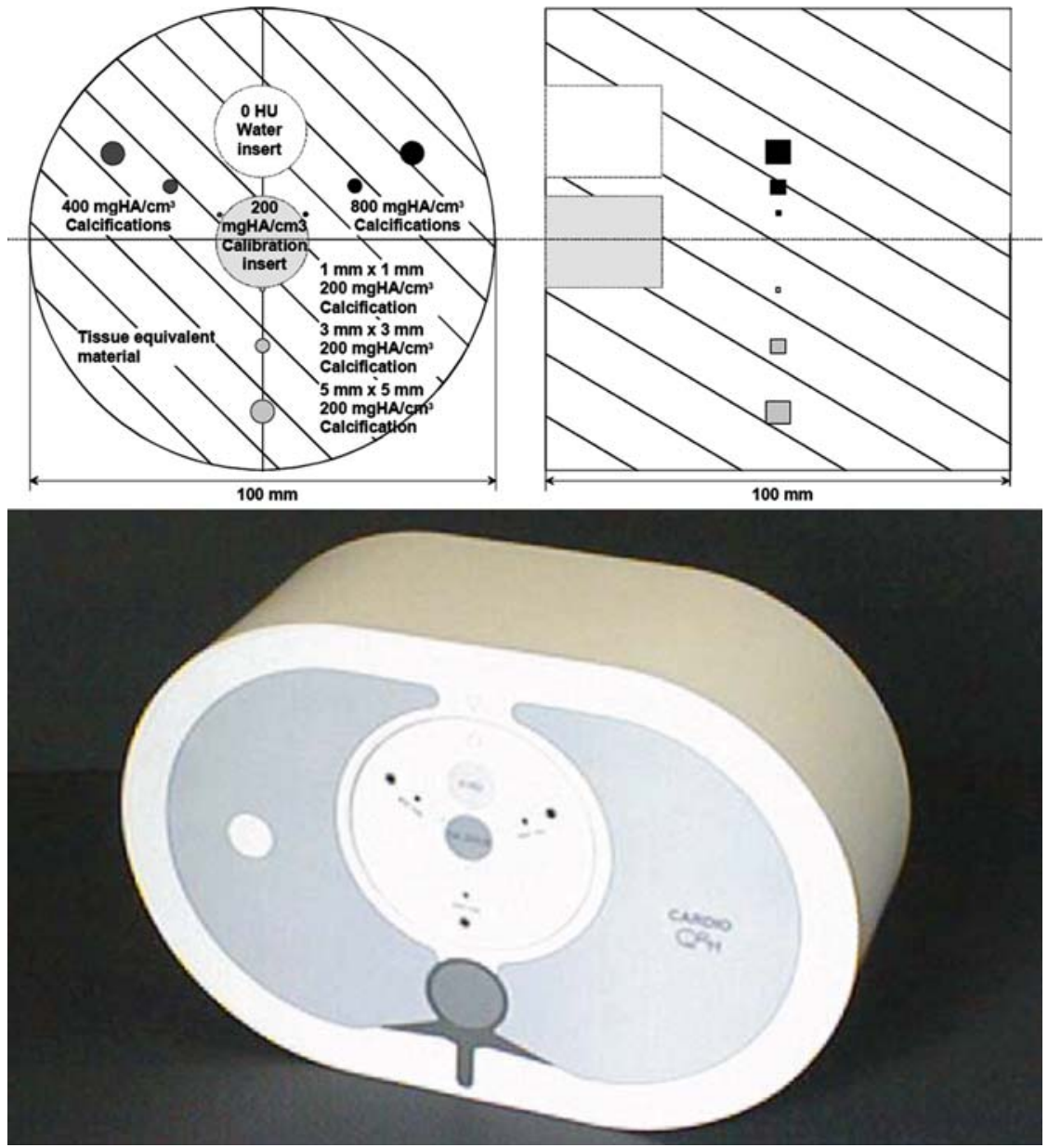

Table 1 Calibration factors $\mathrm{k}$ for all scanners in both sequential and spiral mode

\begin{tabular}{|c|c|c|}
\hline & \multicolumn{2}{|c|}{ Calibration factor $\mathrm{k} \pm \mathrm{SD}$} \\
\hline & Sequential & Spiral \\
\hline System $A_{1}$ & $0.826 \pm 0.007$ & $0.827 \pm 0.008$ \\
\hline System $\mathrm{A}_{2}$ & $0.824 \pm 0.005$ & $0.833 \pm 0.016$ \\
\hline System $\mathrm{A}_{3}$ & $0.832 \pm 0.009$ & $0.827 \pm 0.004$ \\
\hline System $B_{1}$ & $0.703 \pm 0.004$ & $0.720 \pm 0.004$ \\
\hline System $B_{2}$ & $0.725 \pm 0.003$ & $0.732 \pm 0.006$ \\
\hline System $B_{3}$ & $0.723 \pm 0.008$ & $0.722 \pm 0.004$ \\
\hline
\end{tabular}

The variability in percentage $\left(\mathrm{C}_{\mathrm{v}}=\mathrm{SD} / \mathrm{MS}_{\text {mean }}\right.$ *100\%) was determined by calculating the mean mass score $\left(\mathrm{MS}_{\text {mean }}\right)$ and standard deviation (SD) for all individual calcifications using all 30 consecutive scans $(\mathrm{N}=30)$.

For all scanners the smallest calcium inserts of $1 \mathrm{~mm}$ size with a density of 200 and $400 \mathrm{mg} / \mathrm{cm}^{3}$ could not be measured because their HU-values were always less than the threshold $(130 \mathrm{HU})$, therefore these two inserts were omitted from the results yielding seven results for each scan. Subsequently the data were analyzed. (i) Mass scores of identical scanners were compared to each other. (ii) Mass scores were compared to the physical calcium mass. (iii) Mass scores acquired in sequential and spiral scan mode were compared. (iv) Variability of mass scores was assessed.

\section{Statistics}

The scored data were statistically analyzed using SPSS for Windows 14.0 (SPSS Inc, Chicago, USA). A univariate ANOVA $(\alpha=0.05)$ test was used for the comparison between identical scanners. For the comparison between mass score and physical mass a one-sample $t$ test $(\alpha=0.05)$ was used. For the 
comparison of the mass scores between the scan modes two-paired $t$ tests $(\alpha=0.05)$ were used.

\section{Results}

Comparison between identical scanners

Mass scores between the three identical scanners of manufacturer A were similar for almost all calcifications (Table 2). In sequential mode, the ANOVA test showed only significantly different mass scores between the scanners for the high density calcifications of 1 and $3 \mathrm{~mm}$. In spiral scan mode no significantly different mass scores were observed.
Mass score differences were small ranging from 0.1 to $2.9 \mathrm{mg}(1.4-57.4 \%)$ inter-scanner deviation.

Also for manufacturer B mass scores between the three identical scanners were similar for almost all calcifications (Table 3). In sequential scan mode, the mass scores were significantly different for the high density calcifications of 3 and $5 \mathrm{~mm}$ only. In spiral scan mode the $5 \mathrm{~mm}$ high density calcification and the $3 \mathrm{~mm}$ low and medium density calcifications showed significantly different mass scores between the scanners. Mass score differences were small ranging from 0 to $5.8 \mathrm{mg}(0-42.9 \%)$ inter-scanner deviation for manufacturer B.

For both manufacturers, most differences between identical scanners were observed for high density

Table 2 Comparison of mass scores (MS) obtained on systems A in sequential and spiral mode versus physical calcium mass (M)

\begin{tabular}{|c|c|c|c|c|c|}
\hline \multirow{2}{*}{$\begin{array}{l}\text { Calcium cylinder diameter } \\
\text { and density }\left(\mathrm{mg} / \mathrm{cm}^{3}\right)\end{array}$} & \multirow{2}{*}{$\begin{array}{l}\text { Physical calcium } \\
\text { mass } M(\mathrm{mg})\end{array}$} & \multicolumn{3}{|c|}{ Mass scores MS (mg) scanners system A } & \multirow{2}{*}{$\begin{array}{l}\text { ANOVA } \\
\text { system A } \\
P\end{array}$} \\
\hline & & $\mathrm{A}_{1}$ & $\mathrm{~A}_{2}$ & $\mathrm{~A}_{3}$ & \\
\hline \multicolumn{6}{|l|}{ Sequential } \\
\hline Total & 167.8 & $170.3 \pm 3.0^{*}$ & $170.1 \pm 2.8^{*}$ & $173.0 \pm 4.1^{*}$ & $0.001^{\#}$ \\
\hline \multicolumn{6}{|l|}{$5-\mathrm{mm} \varnothing$} \\
\hline 800 & 78.6 & $86.7 \pm 1.2^{*}$ & $86.0 \pm 1.4^{*}$ & $87.2 \pm 3.0^{*}$ & 0.086 \\
\hline 400 & 39.3 & $39.5 \pm 1.1$ & $40.2 \pm 1.3^{*}$ & $40.4 \pm 2.0 *$ & 0.061 \\
\hline 200 & 19.6 & $15.5 \pm 2.5^{*}$ & $15.2 \pm 2.2 *$ & $15.9 \pm 2.4^{*}$ & 0.442 \\
\hline \multicolumn{6}{|l|}{$3-\mathrm{mm} \emptyset$} \\
\hline 800 & 17.0 & $18.1 \pm 0.7^{*}$ & $18.1 \pm 0.6^{*}$ & $18.5 \pm 0.8^{*}$ & $0.035^{\#}$ \\
\hline 400 & 8.5 & $7.5 \pm 0.4^{*}$ & $7.5 \pm 0.6^{*}$ & $7.7 \pm 0.4 *$ & 0.206 \\
\hline 200 & 4.2 & $2.9 \pm 0.6^{*}$ & $2.8 \pm 0.5^{*}$ & $3.0 \pm 0.6^{*}$ & 0.375 \\
\hline \multicolumn{6}{|l|}{$1-\mathrm{mm} \emptyset$} \\
\hline 800 & 0.6 & $0.15 \pm 0.2^{*}$ & $0.25 \pm 0.2^{*}$ & $0.28 \pm 0.2 *$ & $0.012^{\#}$ \\
\hline \multicolumn{6}{|l|}{ Spiral } \\
\hline Total & 167.8 & $165.5 \pm 5.1^{*}$ & $166.7 \pm 7.4$ & $164.0 \pm 5.0 *$ & 0.233 \\
\hline \multicolumn{6}{|l|}{$5-\mathrm{mm} \varnothing$} \\
\hline 800 & 78.6 & $84.3 \pm 3.6^{*}$ & $86.3 \pm 6.0^{*}$ & $85.1 \pm 3.0^{*}$ & 0.213 \\
\hline 400 & 39.3 & $38.5 \pm 2.2$ & $37.6 \pm 2.4^{*}$ & $38.8 \pm 1.8$ & 0.096 \\
\hline 200 & 19.6 & $15.7 \pm 1.6^{*}$ & $15.5 \pm 1.8^{*}$ & $15.2 \pm 1.7^{*}$ & 0.639 \\
\hline \multicolumn{6}{|l|}{$3-\mathrm{mm} \emptyset$} \\
\hline 800 & 17.0 & $17.4 \pm 0.8^{*}$ & $18.0 \pm 1.5^{*}$ & $17.5 \pm 0.9^{*}$ & 0.059 \\
\hline 400 & 8.5 & $7.2 \pm 0.8^{*}$ & $6.9 \pm 0.9^{*}$ & $7.2 \pm 0.8^{*}$ & 0.183 \\
\hline 200 & 4.2 & $2.4 \pm 0.4^{*}$ & $2.3 \pm 0.6^{*}$ & $2.4 \pm 0.4^{*}$ & 0.684 \\
\hline \multicolumn{6}{|l|}{$1-\mathrm{mm} \varnothing$} \\
\hline 800 & 0.6 & $0.10 \pm 0.1^{*}$ & $0.07 \pm 0.1^{*}$ & $0.08 \pm 0.1 *$ & 0.639 \\
\hline
\end{tabular}

*Indicates MS significantly different from M $(P \leq 0.05)$

\# Indicates significantly different MS between scanners $\mathrm{A}_{1}, \mathrm{~A}_{2}$ and $\mathrm{A}_{3}$ 
Table 3 Comparison of mass scores (MS) obtained on systems B in sequential and spiral mode versus physical calcium mass (M)

\begin{tabular}{|c|c|c|c|c|c|}
\hline \multirow{2}{*}{$\begin{array}{l}\text { Calcium cylinder diameter } \\
\text { and density }\left(\mathrm{mg} / \mathrm{cm}^{3}\right)\end{array}$} & \multirow{2}{*}{$\begin{array}{l}\text { Physical calcium } \\
\text { mass M (mg) }\end{array}$} & \multicolumn{3}{|c|}{ Mass scores MS (mg) scanners system B } & \multirow{2}{*}{$\begin{array}{l}\text { ANOVA } \\
\text { system B } \\
P\end{array}$} \\
\hline & & $\mathrm{B}_{1}$ & $\mathrm{~B}_{2}$ & $\mathrm{~B}_{3}$ & \\
\hline \multicolumn{6}{|l|}{ Sequential } \\
\hline Total & 167.8 & $167.7 \pm 6.4$ & $168.8 \pm 3.8$ & $173.2 \pm 3.9^{*}$ & $0.000^{\#}$ \\
\hline \multicolumn{6}{|l|}{$5-\mathrm{mm} \emptyset$} \\
\hline 800 & 78.6 & $84.0 \pm 3.9^{*}$ & $85.0 \pm 2.3^{*}$ & $87.3 \pm 2.6^{*}$ & $0.000^{\#}$ \\
\hline 400 & 39.3 & $39.2 \pm 1.9$ & $39.6 \pm 1.7$ & $40.0 \pm 1.9^{*}$ & 0.189 \\
\hline 200 & 19.6 & $16.6 \pm 2.3^{*}$ & $16.4 \pm 2.5^{*}$ & $17.6 \pm 1.8^{*}$ & 0.100 \\
\hline \multicolumn{6}{|l|}{ 3-mm $\varnothing$} \\
\hline 800 & 17.0 & $17.1 \pm 1.1$ & $17.3 \pm 0.7 *$ & $17.9 \pm 0.7^{*}$ & $0.002^{\#}$ \\
\hline 400 & 8.5 & $7.3 \pm 0.5^{*}$ & $7.5 \pm 0.4^{*}$ & $7.5 \pm 0.6^{*}$ & 0.125 \\
\hline 200 & 4.2 & $2.8 \pm 0.7 *$ & $2.7 \pm 0.6^{*}$ & $2.7 \pm 0.5^{*}$ & 0.770 \\
\hline \multicolumn{6}{|l|}{$1-\mathrm{mm} \emptyset$} \\
\hline 800 & 0.6 & $0.19 \pm 0.1^{*}$ & $0.18 \pm 0.1^{*}$ & $0.20 \pm 0.1^{*}$ & 0.903 \\
\hline \multicolumn{6}{|l|}{ Spiral } \\
\hline Total & 167.8 & $170.8 \pm 2.0^{*}$ & $171.9 \pm 1.7 *$ & $173.4 \pm 3.1^{*}$ & $0.000^{\#}$ \\
\hline \multicolumn{6}{|l|}{$5-\mathrm{mm} \emptyset$} \\
\hline 800 & 78.6 & $85.1 \pm 1.0^{*}$ & $85.8 \pm 1.0^{*}$ & $87.1 \pm 1.3^{*}$ & $0.000^{\#}$ \\
\hline 400 & 39.3 & $40.1 \pm 1.2^{*}$ & $40.7 \pm 1.1^{*}$ & $40.5 \pm 1.2^{*}$ & 0.152 \\
\hline 200 & 19.6 & $17.5 \pm 1.9 *$ & $17.8 \pm 1.4^{*}$ & $17.3 \pm 2.2^{*}$ & 0.603 \\
\hline \multicolumn{6}{|l|}{$3-\mathrm{mm} \emptyset$} \\
\hline 800 & 17.0 & $17.6 \pm 0.5^{*}$ & $17.7 \pm 0.5^{*}$ & $17.7 \pm 0.6^{*}$ & 0.473 \\
\hline 400 & 8.5 & $7.6 \pm 0.3^{*}$ & $7.3 \pm 0.3^{*}$ & $7.7 \pm 0.3^{*}$ & $0.000^{\#}$ \\
\hline 200 & 4.2 & $2.7 \pm 0.5^{*}$ & $2.6 \pm 0.5^{*}$ & $3.0 \pm 0.5^{*}$ & $0.008^{\#}$ \\
\hline \multicolumn{6}{|l|}{$1-\mathrm{mm} \emptyset$} \\
\hline 800 & 0.6 & $0.20 \pm 0.1 *$ & $0.18 \pm 0.1 *$ & $0.25 \pm 0.1^{*}$ & 0.100 \\
\hline
\end{tabular}

*Indicates MS significantly different from M $(P \leq 0.05)$

\# Indicates significantly different MS between scanners $\mathrm{B}_{1}, \mathrm{~B}_{2}$ and $\mathrm{B}_{3}$

calcifications. For the total mass scores inter-scanner differences ranged from 2.6 to $5.8 \mathrm{mg}(1.5-3.4 \%)$. Between the identical scanners of manufacturer A fewer mass score differences were observed than between the identical scanners of manufacturer B (3 vs. 7).

Acquired mass score compared to physical calcium mass

Mass scores were significantly $(P \leq 0.05)$ different from the physical mass for all scanners and almost all calcifications (Tables 2, 3). Deviation of the mass scores from the physical mass; however, was consistent for all scanners (Fig. 2). Severe underestimation was found for the $1 \mathrm{~mm}$ calcification, ranging from 103 to $710 \%$ for all scanners and both manufacturers. The $3 \mathrm{~mm}$ calcifications of low and medium density were on average underestimated by 57 and $15 \%$ respectively and the high density calcification was overestimated $4 \%$. The $5 \mathrm{~mm}$ low density calcification was underestimated by all scanners $(-20 \%)$ and the medium density calcification was overestimated by $1.7 \%$ except for scanners $\mathrm{A}$ in spiral mode which showed an underestimation of $2.6 \%$. The high density calcification of $5 \mathrm{~mm}$ was overestimated by all scanners $(+8.4 \%)$.

The total physical mass $(167.8 \mathrm{mg})$ was significantly overestimated $(+2.3 \%)$ by system $\mathrm{A}$ and two scanners of system B when sequential scan mode was 

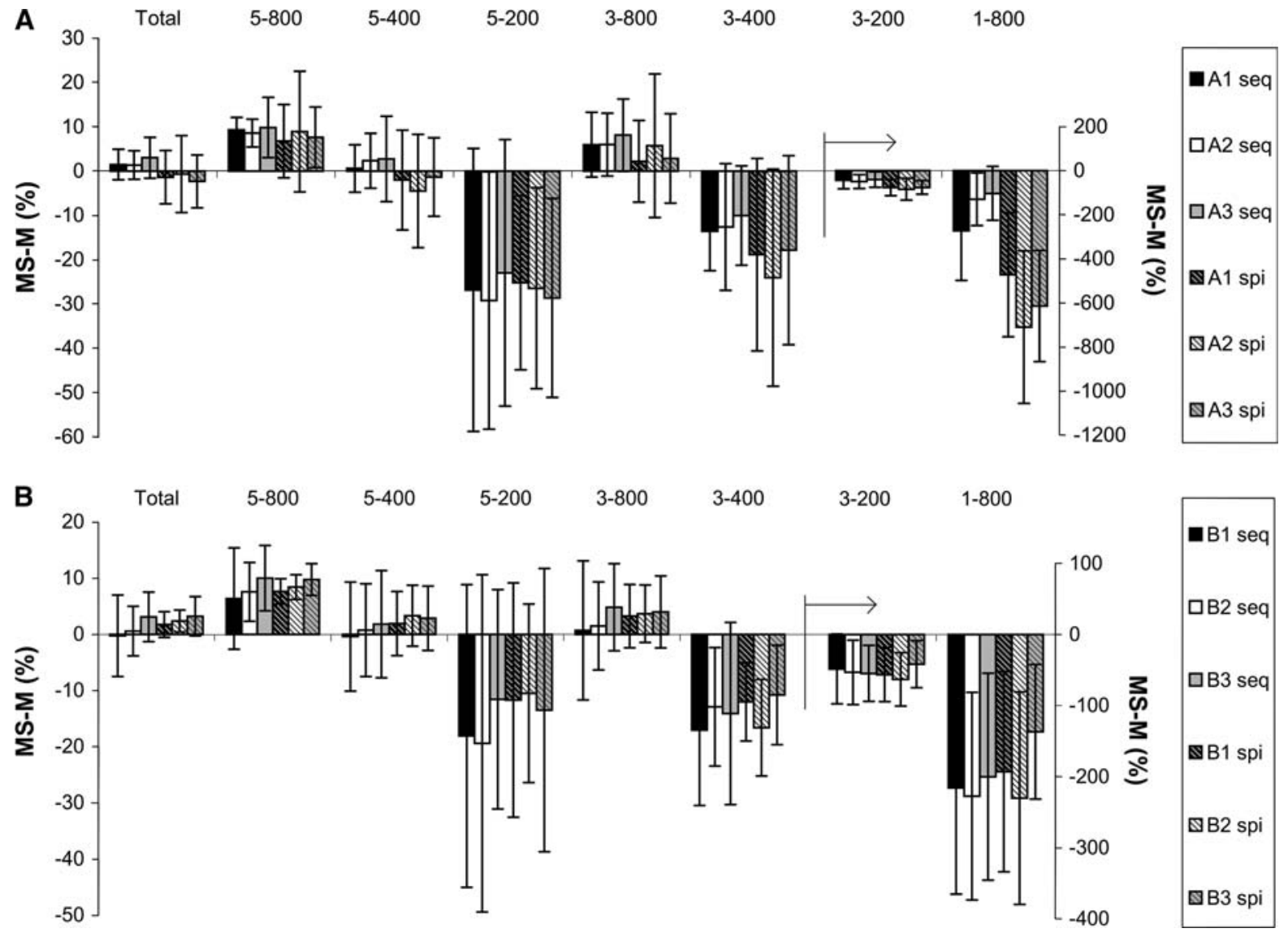

Fig. 2 Difference (\%) observed between mass scores (MS) and physical mass (M) with 95\% confidence bands. Mass scores were obtained on three 64-slice MDCT systems of manufacturer A (a) and three of manufacturer B (b) in sequential and spiral scan mode

used. In spiral mode a significant underestimation $(-1.8 \%)$ was found for two scanner of system A and a significant overestimation $(+2.5 \%)$ for system B.

The average total mass scores were different between manufacturer A and $\mathrm{B}$. In sequential mode the average total mass scores were significantly $(P \leq 0.028)$ higher for scanners A $(171.1 \mathrm{mg})$ compared to scanners B $(169.9 \mathrm{mg})$. In spiral mode the average total mass scores were significantly $(P \leq 0.001)$ lower for scanners A $(165.4 \mathrm{mg})$ compared to scanners B $(172.1 \mathrm{mg})$.

Sequential versus spiral scan mode

Scan mode had a different effect on the mass scores for each manufacturer. For system $\mathrm{A}$, the average total mass scores were significantly $(P \leq 0.026)$ higher in sequential mode $(171.1 \mathrm{mg})$ compared to spiral mode $(165.4 \mathrm{mg})$. System B showed the opposite effect where the average total mass scores were significantly $(P \leq 0.017)$ lower in sequential mode $(169.8 \mathrm{mg})$ compared to spiral mode $(172.0 \mathrm{mg})$. Scanner $\mathrm{B}_{3}$ did not show significant differences between the scan modes $(P=0.786)$.

For system A using spiral scan mode no differences were found between identical scanners, whereas a few differences were found using sequential mode. For system B the scan mode did not affect the number of different mass scores between identical scanners.

The variability of the total mass scores was lower in sequential mode compared to spiral mode for system A whereas for system B the variability was lower in spiral mode (Fig. 3).

Variability of mass scores

The variability of the mass scores increased when the density of the calcifications decreased (Fig. 4). Large 


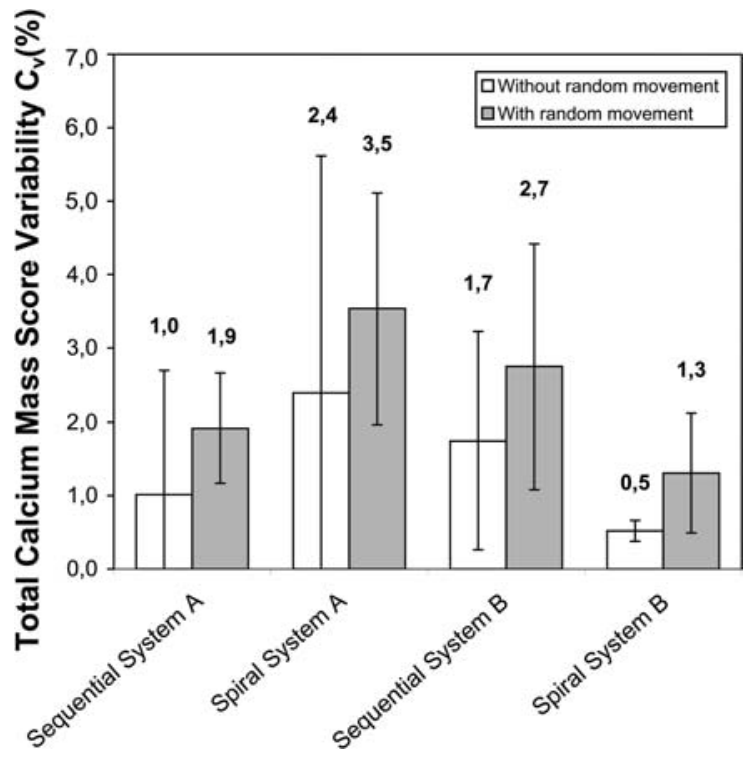

Fig. 3 Variability in percentage $\left(C_{v}\right)$ of the total mass score averaged over the three scanners of system $\mathrm{A}$ and $\mathrm{B}$ in sequential and spiral scan mode. Results are shown with and without random movement of the phantom on the table

$5 \mathrm{~mm}$ calcifications yielded lower mass score variabilities than the smaller $3 \mathrm{~mm}$ calcifications, ranging from 1.1 to $25.5 \%$. The smallest calcification of $1 \mathrm{~mm}$ showed the highest variability (48-177\%).

The variability of the total mass scores was $1.9 \%$ for system $\mathrm{A}$ in sequential mode and $3.5 \%$ in spiral scan mode (Fig. 4). The variability of the total mass scores was $2.7 \%$ for system B in sequential mode and $1.3 \%$ in spiral scan mode. Additionally, the average variability of the total mass scores without random movement of the phantom was lower than when movement was added to the experiment (Fig. 3).

\section{Discussion}

Comparison between identical scanners

In this study we investigated whether mass scores are comparable or differ between identical scanners of the same manufacturer. We found that mass scores between identical scanners were similar for almost all calcifications. In a few cases the mass scores were significantly different between identical scanners, in particular for high density calcifications. However, in all cases the absolute differences between the mean mass scores of the identical scanners were small and for the total mass scores the differences ranged from 1.5 to $3.4 \%$. These differences are in the same order of magnitude compared to the mass score variability as measured for each scanner separately (1.3-3.5\%). Calibration of the measurement guaranteed that the HU-scales of all scanners were fixed to known calcium mass densities and that consequently any calcium mass off-sets between the scanners were cancelled. It is therefore likely that the small differences in mass scores between identical scanners are the result of the displacement of the phantom on the table and table movement variations [17].

In a previous study no differences were observed between scanners of the same manufacturer when the Agatston and volume score algorithms were used [18]. Variability of the Agatston and volume score is relatively high compared to the mass score variability [9-13]. The probability to detect significant differences between groups increases when the variability of the measurement decreases. This explains why we found some significantly different mass scores between scanners in a few cases when the mass score algorithm was used.

Acquired mass score compared to physical calcium mass

Next to the similarity of mass scores between identical scanners we assessed the accordance of the mass scores with the physical mass. We found that overall all scanners showed mass scores in good agreement with the physical calcium mass. Low density calcifications tended to be underestimated and high density calcifications were overestimated. However, calcium plaques in vivo will usually be constituted out of mixed density components and the total mass score is usually being used as a measure for risk stratification, therefore on average overestimation will be compensated by underestimation. Very small calcifications were severely underestimated by all scanners as a result of the partial volume effect in combination with an improper scoring threshold (default $130 \mathrm{HU}$ ). This can jeopardize the discrimination between a zero-calcium score and initial coronary calcification. A dynamic scoring threshold, dependent on the calcium density, is expected to improve the agreement between mass scores and the physical mass [13, 19-21]. The 

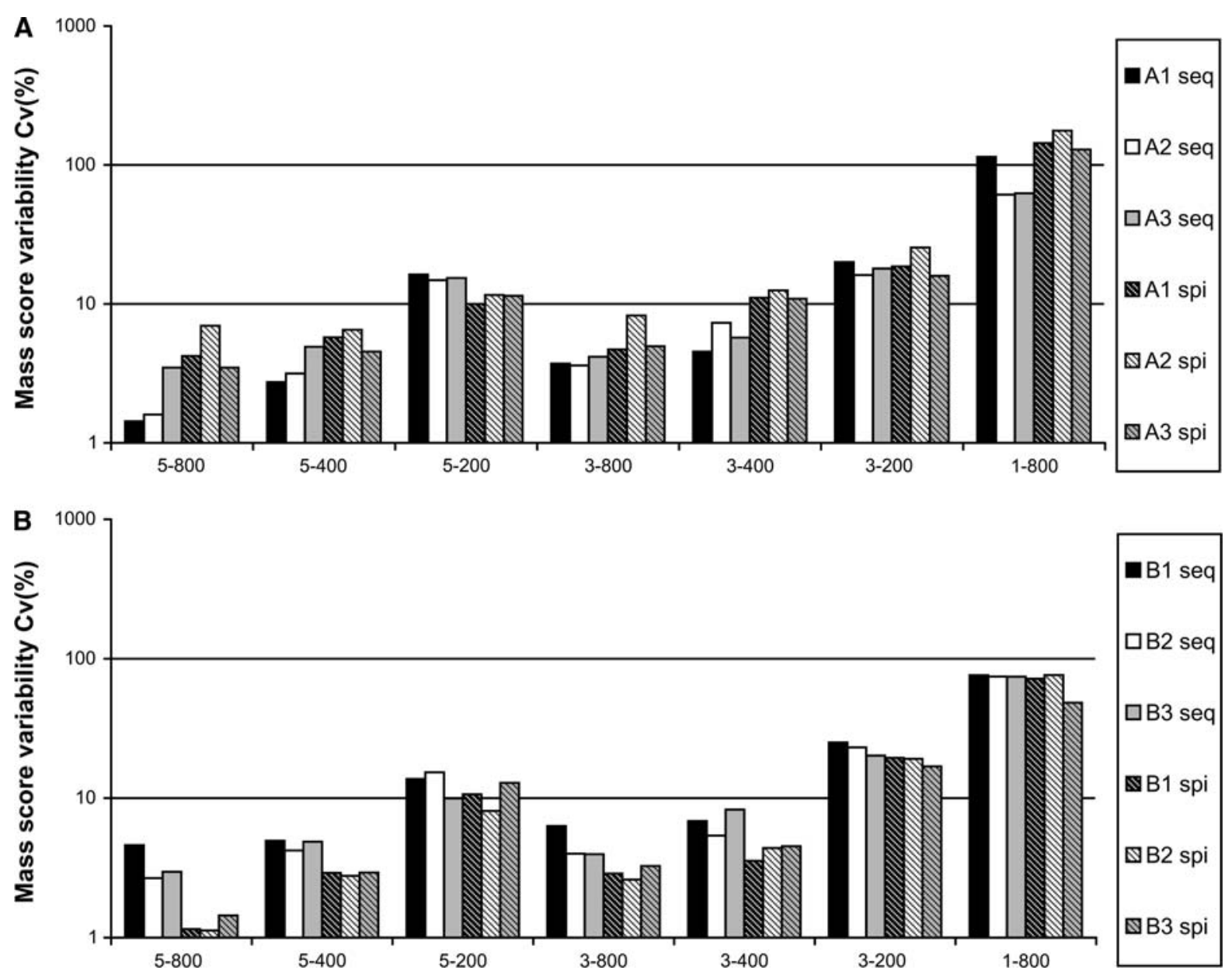

Fig. 4 Variability $\left(\mathrm{C}_{\mathrm{v}}\right)$ of measured mass scores of the seven calcifications. Mass scores were obtained on three 64-slice MDCT systems of manufacturer A (a) and three of manufacturer B (b) in sequential and spiral scan mode

amount of underestimation of very small calcifications however, was consistent between all scanners.

Sequential versus spiral scan mode

Both sequential and spiral scan modes were used to determine the effect of the acquisition mode on the mass scores obtained on identical scanners. It was observed that scan mode had a different effect on the mass scores for each manufacturer. Total mass scores were significantly different between identical scanners of system A when sequential scan mode was used; however, no significant differences were found using the spiral scan mode. For system B the total mass scores were significantly different in both scan modes.

Furthermore, system A showed higher mass scores in sequential mode compared to spiral mode, whereas for system B the opposite was observed. The latter can be due to the higher tube current in spiral scan mode, however, it was also shown that tube current does not have a significant effect on HU-values and consequently mass scores are not expected to be dependent on tube current [22]. Furthermore, system A showed lower mass scores in spiral mode.

The observed differences between the scan modes indicate that the scan mode is an important aspect in calcium mass quantification and that the recommended scan mode strongly depends on the scanner manufacturer.

Variability of mass scores

The precision of mass scores was assessed by determining the mass score variability of repeated 
scans. Besides the dependence of absolute mass scores on the scan mode we found that the variability of the mass scores is also dependent on the scan mode. The mass score variability increased almost twofold from sequential (1.9\%) to spiral scan mode (3.5\%) which is similar to a previous study [23]. Remarkably, for system B the total mass score variability decreased twofold from sequential $(2.7 \%)$ to spiral scan mode $(1.3 \%)$ which was also found in another study [24]. In addition, recently it was shown that the mass score variability is smallest for high density calcifications, which is confirmed by our findings [25].

Variability of the mass scores is related to the variations in HU-values between the scans, which are mainly due to table movement and displacement of the phantom on the table. The average variability of the mass scores without displacement of the phantom was lower than when random displacement was added to the experiment. Without displacing the phantom on the table the mass score variability was still in between 0.5 and $2.4 \%$. From this it can be concluded that the variability of mass scores is affected for a large part by table movement. This is confirmed in a previous study that showed that a large part of score variability and mass score variability is due to the variations in scan starting position [17].

\section{Limitations}

In this study we simulated the random positioning of the coronaries by performing a random translation and rotation of the phantom in between each consecutive scan. The variability resulting from this procedure is a significant fraction of the overall variability of realistically moving coronaries. However, to obtain a mass score variability even more in agreement with in vivo conditions, one has to take realistic heart motion into account. It was recently shown that Agatston, volume and mass scores are strongly heart rate dependent [26, 27]. In vivo, coronaries can show average velocities of $69.5 \mathrm{~mm} /$ $\mathrm{s}$ (RCA), $22.4 \mathrm{~mm} / \mathrm{s}$ (LAD) and $48.4 \mathrm{~mm} / \mathrm{s}$ (CCA) [28]. It is therefore expected that the variability of mass scores will increase when realistic heart movement is included, and that the few remaining differences observed between identical scanners in this study will become non-significant and the mass scores obtained on separate scanners are comparable.
Clinical applicability

The variability in mass scores found in this nonmoving phantom study was relatively small, compared to the reproducibility reported for the calcium score according to Agatston. The Agatston score is still mainly used in clinical practice, also for determining change in extent of coronary calcium on sequential scans. The percentage of change in Agatston score that is considered significant, taking into account interscan variability, ranged in one clinical study from 24 to $190 \%$ [17]. The differences in mass scores we found for identical scanners are much smaller (0-57.4\%). If studies in moving heart phantoms and in vivo confirm our results, change in coronary calcification can be more accurately assessed by applying the mass score. No repeatability limits are yet available for the mass score. These will have to be determined from large, population-based studies.

\section{Conclusions}

Assessment of coronary calcium mass quantified by the mass score algorithm showed that mass scores are comparable between identical 64-slice CT scanners. Identical 64-slice CT scanners of the same manufacturer can be used in follow-up studies when calcium mass scores are used to predict the risk of myocardial infarction. This study also showed the need for using the same scan mode in follow-up studies. Furthermore, for all systems significant differences were found between mass scores and the physical calcium mass; however, the differences were relatively small and consistent.

Open Access This article is distributed under the terms of the Creative Commons Attribution Noncommercial License which permits any noncommercial use, distribution, and reproduction in any medium, provided the original author(s) and source are credited.

\section{References}

1. Agatston AS, Janowitz WR, Detrano R et al (1990) Quantification of coronary artery calcium using ultrafast computed tomography. J Am Coll Cardiol 15:827-832

2. Vliegenthart R, Oudkerk M, Witteman JC (2005) Coronary calcification improves cardiovascular risk prediction in the elderly. Circulation 112(4):572-577 
3. Detrano R, Guerci AD, Kronmal RA et al (2008) Coronary calcium as a predictor of coronary events in four racial or ethnic groups. N Engl J Med 358(13):1336-1345

4. Geluk CA, Dikkers R, Zijlstra F et al (2008) Measurement of coronary calcium scores by electron beam computed tomography or exercise testing as initial diagnostic tool in low-risk patients with suspected coronary artery disease. Eur Radiol 18(2):244-252

5. Arad Y, Spadaro LA, Goodman K et al (2000) Prediction of coronary events with electron beam computed tomography. J Am Coll Cardiol 36(4):1253-1260

6. Callister TQ, Cooil B, Raggi P et al (1998) Coronary artery disease: improved reproducibility of calcium scoring with an electron-beam CT volumetric method. Radiology 208: 807-814

7. Detrano R, Kang X, Nickerson S et al (1994) Accuracy of quantifying coronary hydroxyapatite with electron beam tomography. Investig Radiol 29(8):733-738

8. Ulzheimer S, Kalender WA (2003) Assessment of calcium scoring performance in cardiac computed tomography. Eur Radiol 13(3):484-497

9. McCollough $\mathrm{CH}$, Ulzheimer S, Kalender WA et al (2007) Coronary artery calcium: a multi-institutional, multimanufacturer international standard for quantification at cardiac CT. Radiology 243(2):527-538

10. Hoffmann U, Siebert U, O'Donnell CJ et al (2006) Evidence for lower variability of coronary artery calcium mineral mass measurements by multi-detector computed tomography in a community-based cohort-consequences for progression studies. Eur J Radiol 57(3):396-402

11. Horiguchi J, Shen Y, Ito K et al (2005) Electron beam CT versus 16-MDCT on the variability of repeated coronary artery calcium measurements in a variable heart rate phantom. AJR Am J Roentgenol 185(4):995-1000

12. Hong C, Bae KT, Zhu F et al (2003) Coronary artery calcium quantification at multi-detector row $\mathrm{CT}$ : influence of heart rate and measurement methods on interacquisition variability initial experience. Radiology 228(1):95-100

13. Hong C, Bae KT, Pilgram TK (2003) Coronary artery calcium: accuracy and reproducibility of measurements with multi-detector row CT-assessment of effects of different thresholds and quantification methods. Radiology 227(3):795-801

14. Detrano RC, Anderson M, Bild DE (2005) Coronary calcium measurements: effect of CT scanner type and calcium measure on rescan reproducibility-MESA study. Radiology 236(2):477-484

15. Chung H, McClelland RL, Budoff MJ (2008) Repeatability limits for measurement of coronary artery calcified plaque with cardiac CT in the multi-ethnic study of atherosclerosis. AJR Am J Roentgenol 190(2):W87-W92

16. Sevrukov AB, Bland JM, Kondos GT (2005) Serial electron beam CT measurements of coronary artery calcium: has your patient's calcium score actually changed? AJR Am J Roentgenol 185(6):1546-1553
17. Rutten A, Isgum I, Prokop M (2008) Coronary calcification: effect of small variation of scan starting position on Agatston, volume, and mass scores. Radiology 246(1): 90-98

18. Greuter MJW, Dijkstra H, Groen JM et al (2007) 64 Slice MDCT generally underestimates coronary calcium scores as compared to EBT: a phantom study. Med Phys 34(9):3510-3519

19. Hong C, Pilgram TK, Bae KT (2003) Improving mass measurement of coronary artery calcification using threshold correction and thin collimation in multi-detector row computed tomography: in vitro experiment. Acad Radiol 10(9):969-977

20. Raggi P, Callister TQ, Cooil B (2002) Calcium scoring of the coronary artery by electron beam CT: how to apply an individual attenuation threshold. AJR Am J Roentgenol 178(2):497-502

21. Groen JM, Dijkstra H, Greuter MJ, Oudkerk M (2009) Threshold adjusted calcium scoring using CT is less susceptible to cardiac motion and more accurate. Med Phys 36(2):438-446

22. Birnbaum BA, Hindman N, Lee J, Babb JS (2007) Multidetector row CT attenuation measurements: assessment of intra- and interscanner variability with an anthropomorphic body CT phantom. Radiology 242(1):109-119

23. Rosol M, Sachdev K, Hoffmann U et al (2006) A novel model to test accuracy and reproducibility of MDCT scan protocols for coronary calcium in vivo. Int $\mathrm{J}$ Cardiovasc Imaging 22(1):111-118

24. Horiguchi J, Kiguchi M, Fujioka C, Arie R, Shen Y, Sunasaka K, Kitagawa T, Yamamoto H, Ito K (2008) Variability of repeated coronary artery calcium scoring and radiation dose on 64- and 16-slice computed tomography by prospective electrocardiographically-triggered axial and retrospective electrocardiographically-gated spiral computed tomography: a phantom study. Acad Radiol 15(8): 958-965

25. Thomas CK, Muhlenbruch G, Mahnken AH et al (2006) Coronary artery calcium scoring with multislice computed tomography: in vitro assessment of a low tube voltage protocol. Investig Radiol 41(9):668-673

26. Groen JM, Greuter MJ, Schmidt B, Suess C, Vliegenthart R, Oudkerk M (2007) The influence of heart rate, slice thickness, and calcification density on calcium scores using 64-slice multidetector computed tomography: a systematic phantom study. Investig Radiol 42(12):848-855

27. Groen JM, Greuter MJ, Vliegenthart R, Suess C, Schmidt B, Zijlstra F, Oudkerk M (2008) Calcium scoring using 64-slice MDCT, dual source CT and EBT: a comparative phantom study. Int J Cardiovasc Imaging 24(5):547-556

28. Achenbach S, Ropers D, Holle J, Muschiol G, Daniel WG, Moshage W (2000) In-plane coronary arterial motion velocity: measurement with electron-beam CT. Radiology 216(2):457-463 